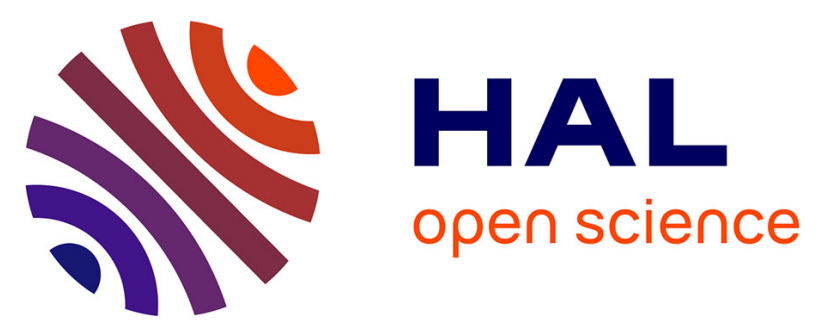

\title{
Infrared spectroscopy quantification of functional carbon groups in kerogens and coals: A calibration procedure
} van T.H. Phan, Eric Quirico, Pierre Beck, Yann Le Brech, Lora Jovanovic,

Corentin Le Guillou, Sylvain Bernard, Lydie Bonal, Nathalie Carrasco, Thomas Gautier, et al.

\section{To cite this version:}

van T.H. Phan, Eric Quirico, Pierre Beck, Yann Le Brech, Lora Jovanovic, et al.. Infrared spectroscopy quantification of functional carbon groups in kerogens and coals: A calibration procedure. Spectrochimica Acta Part A: Molecular and Biomolecular Spectroscopy [1994-... , 2021, 259 (October), pp.119853. 10.1016/j.saa.2021.119853 . insu-03208201

\section{HAL Id: insu-03208201 \\ https://hal-insu.archives-ouvertes.fr/insu-03208201}

Submitted on 12 Oct 2021

HAL is a multi-disciplinary open access archive for the deposit and dissemination of scientific research documents, whether they are published or not. The documents may come from teaching and research institutions in France or abroad, or from public or private research centers.
L'archive ouverte pluridisciplinaire HAL, est destinée au dépôt et à la diffusion de documents scientifiques de niveau recherche, publiés ou non, émanant des établissements d'enseignement et de recherche français ou étrangers, des laboratoires publics ou privés. 


\section{Infrared spectroscopy quantification of functional carbon}

groups in kerogens and coals: A calibration procedure

Van T.H. Phan ${ }^{1}$, Eric Quirico ${ }^{1}$, Pierre Beck ${ }^{1}$, Yann Le Brech ${ }^{2}$, Lora Jovanovic ${ }^{3}$, Corentin Le Guillou$^{4}$, Sylvain Bernard ${ }^{5}$, Lydie Bonal ${ }^{1}$, Nathalie Carrasco ${ }^{3}$, Thomas Gautier ${ }^{3}$, Jesus Raya ${ }^{6}$

${ }^{1}$ Institut de Planétologie et d'Astrophysique de Grenoble (IPAG), Université Grenoble 9 Alpes/CNRS-INSU, UMR 5274, Grenoble F-38041,

${ }^{2}$ LRGP, CNRS, Université de Lorraine, 1 rue Grandville 54000 Nancy, France

${ }^{3}$ CNRS/INSU, LATMOS-IPSL, Guyancourt F-78280, France

${ }^{4}$ Unité Matériaux et Transformations, UMET, UMR-CNRS 8207, Université de Lille, France

${ }^{5}$ Muséum National d'Histoire Naturelle, Sorbonne Université, CNRS UMR 7590, IRD, Institut de Minéralogie, de Physique des Matériaux et de Cosmochimie, Paris, France

${ }^{6}$ ICS, CNRS, Université de Strasbourg, BP 296 R8 670008 Strasbourg

\section{Corresponding author:}

19 Van Thi Hai Phan

Email: thi-hai-van.phan@univ-grenoble-alpes.fr 


\section{ABSTRACT}

The determination of the abundances of the $\mathrm{CH}_{x}, \mathrm{C}=\mathrm{O}$ and aromatic groups in chondritic Insoluble Organic Matter (IOM) and coals by Infrared (IR) spectroscopy is a challenging issue, due to insufficient knowledge on the absorption cross-sections and their sensitivity to the molecular environment. Here, we report on a calibration approach based on a ${ }^{13} \mathrm{C}$ synthetic model material, whose composition was unambiguously determined by Direct-Pulse/Magic Angle Spinning Nuclear Magnetic Resonance (DP/MAS NMR). Ratios of the cross-sections of the $\mathrm{CH}_{\mathrm{x}}, \mathrm{C}=\mathrm{O}$ and aromatic groups have been determined, and the method has been applied to IOM samples extracted from four chondrites as Orgueil (CI), Murchison (CM), Tagish Lake (C2-ungrouped) and EET 92042 (CR), and to a series of coals. The estimate of the aliphatic to aromatic carbon ratio ( $\mathrm{n}_{\mathrm{CH}} / \mathrm{n}_{\mathrm{Aro}}$ ) in IOM samples from Orgueil, Murchison and Tagish Lake chondrites is in good agreement with Single-Pulse/NMR estimates earlier published, and is lower by a factor 1.3 in the case of the CR chondrite EET 92042 (but error bars overlap). In contrast, the aliphatic to carbonyl ratio $\left(\mathrm{n}_{\mathrm{CHx}} / \mathrm{n}_{\mathrm{C}=\mathrm{O}}\right)$ is overestimated for the four chondrites. These discrepancies are likely due to the control of the absorption cross-section of the $\mathrm{C}=\mathrm{O}$ and $\mathrm{C}=\mathrm{C}$ bonds by the local molecular environment. Regarding coals, the use of published NMR analyses has brought to light that the integrated cross-section ratio AcHx/AAro varies with the vitrinite reflectance over an order of magnitude. Here as well, the local oxygen speciation plays a critical control on AAro, which decreases with increasing the vitrinite reflectance. We provide an analytical law that links $A_{C H x} / A_{A r o}$ and vitrinite reflectance, which will allow the determination of ncHx/nAro for any coal sample, provided its vitrinite reflectance is known.

Keywords: Infrared spectroscopy, Nuclear Magnetic Resonance (NMR), Kerogens, Coals, Insoluble Organic Matter, Chondrites, Composition (1)

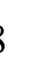




\section{INTRODUCTION}

Infra-Red (IR) spectroscopy is a non-destructive technique well suited to characterize complex insoluble organic compounds in meteorites and coals. It provides an estimation of organic composition based on the integrated absorbance of the main functional groups (e.g., alkyl $\mathrm{CH}, \mathrm{CH}_{2}$ and $\mathrm{CH}_{3}$, aromatic $\mathrm{C}=\mathrm{C}$ and $\mathrm{C}-\mathrm{H}$, carbonyl/carboxylic $\mathrm{C}=\mathrm{O}$, hydroxyl $-\mathrm{OH}$ ) and tracers of the chemical and structural evolution during diagenesis and catagenesis ( $\mathrm{Li}$ et al., 2016; Lis et al., 2005a; Painter et al., 1981; Petersen et al., 2008; Sun, 2005; Chen et al., 2012; Xueqiu et al., 2017). In addition, micro-FTIR, and more recently, Atomic force microscopybased infrared spectroscopy (AFM-IR), has offered new perspectives to collect spatially resolved IR spectra, thereby identifying chemical heterogeneities at the submicrometric scale (Yang et al., 2017; Jakob et al., 2019; Abarghani et al., 2020; Jubb et al., 2020). These techniques have been proved valuable for the characterization of precious extraterrestrial micrometric grains, like Interplanetary Dust Particles (IDPs), Antarctic micro-meteorites and matrix grains extracted from rare primitive chondrites (Battandier et al., 2018; Beck et al., 2010; Dartois et al., 2004; Kebukawa et al., 2018; Mathurin et al., 2019; Orthous-Daunay et al., 2013; Quirico et al., 2018; Quirico et al., 2014). Although the assignment of the main vibration bands has reached a consensus, quantifying the exact proportions of functional groups based on IR signal remains difficult (Painter et al., 1981; delaRosa et al., 1992). Indeed, only a few absorption cross-sections have been published so far (Sandford et al., 1991; Dartois et al., 2004; Russell et al., 2009; Takahama et al., 2013). In addition, due to the complex structure of kerogens and coals, a given chemical group exists within different molecular environments, and is difficult to reside in various frequency shifts, inhomogeneous broadening and variability of the absorption cross-section values (Craddock et al., 2015).

Here, we report the determination of the absorption cross-sections of the alkyl $\left(\mathrm{CH}_{\mathrm{x}}\right)$, carbonyl $(\mathrm{C}=\mathrm{O})$ and aromatic $(\mathrm{C}=\mathrm{C})$ groups based on a $\mathrm{H}^{13} \mathrm{CO}$ synthetic kerogen analogue (named $\mathrm{H}^{13} \mathrm{CO}$-tholins), whose composition is unambiguously determined by DP/MAS (Direct Polarization/Magic Angle Spinning) Nuclear Magnetic Resonance and XANES (X-ray Absorption at Near-Edge Spectroscopy) at the C K-edge. The use of a ${ }^{13} \mathrm{C}$ sample (e.g. synthesized $\mathrm{H}^{13} \mathrm{CO}$-tholins) overcomes the critical bias of undetected carbons encountered in solid-state ${ }^{13} \mathrm{C}$ NMR analysis. These absorption cross-sections have been applied to a series of infrared spectra of kerogens extracted from primitive chondrites, and to a series of coals ranging a broad range of maturity. As the relative abundances of $\mathrm{C}=\mathrm{O}, \mathrm{C}=\mathrm{C}$ and $\mathrm{CH}_{\mathrm{x}}$ groups in these samples are reported in several publications, the calibration protocol could be tested, and we further discuss the dependency of the cross-sections with the chemical structure.

\section{MATERIALS AND METHODS}

\subsection{Sample selection and preparation}

\subsubsection{Production of IOM analogues from HCO-tholins}


HCO-tholins samples are polymeric disordered carbonaceous materials that were synthesized in the PAMPRE experimental reactor located at LATMOS (Guyancourt, France) (Alcouffe et al., 2010; Szopa et al., 2006). This setup consists in a radio-frequency capacitively coupled plasma (RF CCP) confined within a cylindrical cage, filled with an initial gaseous mixture made of $7 \%$ of $\mathrm{CH}_{4}, 3 \%$ of $\mathrm{CO}$ and $90 \%$ of Ar gas, with a $3 \mathrm{sccm}$ flux under the total pressure of 0.43 mbar. Two types of $\mathrm{H}^{12} \mathrm{CO}$ and $\mathrm{H}^{13} \mathrm{CO}$ tholins were produced from natural ${ }^{12} \mathrm{C}$ rich and ${ }^{13} \mathrm{C}$ - rich gases (purity $>99.5 \%$ ), provided by Eurisotop (Saclay, France). The power delivered by the generator was maintained within 11-12 W. Grains were formed within the plasma and kept under electrostatic levitation, and grew up until they deposited into a glass vessel surrounding the confining cage of the plasma. Experiments were run daily (8-10 h) during working hours for safety. The temperature of the electrode was $21^{\circ} \mathrm{C}$ when starting the discharge, and $45^{\circ} \mathrm{C}$ when switching it off in the evening. At total, about $30 \mathrm{mg}$ of $\mathrm{H}^{13} \mathrm{CO}$-tholins were obtained in 63 hours, and $130 \mathrm{mg}$ of $\mathrm{H}^{12} \mathrm{CO}$-tholins in 98 hours. The production yield, 0.50 to 1.3 mg.hour ${ }^{-1}$, was much lower compared to tholins formed from $\mathrm{N}_{2}$ : $\mathrm{CH}_{4}$ mixtures (Sciamma-O'Brien et al., 2010). The $\mathrm{H}^{13} \mathrm{CO}$-tholin was composed of a dominant yellow, finegrained powder and a less abundant population of dark platelets (Fig. S-1). In comparison, the $\mathrm{H}^{12} \mathrm{CO}$-tholins did not contain any dark grains. The black platelets were constituted by direct deposition on the grid, which eventually fell and mixed with the dusty tholins. Infrared spectroscopy has shown that these two populations were chemically similar (Fig. S-2). Finally, the differences in the color of the grains are probably due to the larger thickness of the platelets.

Analogues of kerogens and coals were obtained from the carbonization of $\mathrm{H}^{13} \mathrm{CO}$-tholins with the elemental composition revealed in Table S-1 (Supporting Information). To select optimal conditions to get the good analogues, preliminary heating experiments were run at 200 ${ }^{\circ} \mathrm{C}, 300{ }^{\circ} \mathrm{C}, 350{ }^{\circ} \mathrm{C}, 400{ }^{\circ} \mathrm{C}, 450{ }^{\circ} \mathrm{C}, 500{ }^{\circ} \mathrm{C}$ and $550{ }^{\circ} \mathrm{C}$, with a tubular furnace maintained under secondary vacuum ( $\sim 10^{-6}$ mbar). Small grains of samples were selected and pressed between two diamond windows and put into a ceramic crucible that was placed at the middle of the tube. For each experiment, the setting temperature was maintained during $1 \mathrm{~h}$, and the tube was then let cool down freely for 3-6 hours, depending on the initial temperature setting. Infrared spectra of the heated samples were collected and compared to spectra of Insoluble Organic Matter (IOM) samples extracted from primitive chondrites (Fig. S-2). Finally, we select a temperature of $400^{\circ} \mathrm{C}$ (for extensive details on the procedure, see Supporting Information). The final carbonization of HCO-tholins was then operated with this set temperature, using a ceramic crucible filled with 5-10 mg of the powdered samples. We used an anti-static gun (Zerostat, Sigma-Aldrich) to limit sample loss due to static electricity.

\subsubsection{Coal and IOM samples}

A suite of coal samples with a mean maximum vitrinite reflectance $\left(\mathrm{R}_{0}\right)$ ranging $0.33-$ 2.8\% were purchased from the Penn State University coal sample Bank (http://www.energy.psu.edu) (Table S-2). The IR spectra of IOMs extracted from primitive chondrites have been earlier published in Orthous-Daunay et al. (2013), including IOMs from Orgueil (CI1), Murchison (CM2), EET92042 (CR2) and Tagish Lake (C2-ung), while the IR 


\subsection{Analytical methods}

142

143

144

145

146

147

148

149

150

151

152

153

154

155

156

157

158

159

160

161

162

163

164

165

166

167

168

169

170

171

172

173

174

175

176

177

178

179

180

\subsubsection{FTIR measurements}

The FTIR instrument used was a Bruker Hyperion 3000 infrared microscope, equipped with liquid nitrogen cooled-mercury cadmium telluride (MCT) detector. Micro-FTIR spectra were collected in the range of $4000-700 \mathrm{~cm}^{-1}$ with a spectral resolution of $4 \mathrm{~cm}^{-1}$. No samples required more than 200 scans ( $5 \mathrm{~min}$ ) to get a satisfying signal-to-noise ratio. All measurements were done using an environmental cell with an optical path through two ZnS windows and pumped to $\sim 10^{-6}$ mbar (Beck et al., 2010). The sample (e.g., tholins, coal) was deposited onto a type IIa synthetic diamond window (3 mm diameter, $500 \mu \mathrm{m}$ thick) and crushed with a similar window to have a thin and flat sample. The diamond window holding the crushed sample was inserted in the cell, pumped, and the window was heated at $80^{\circ} \mathrm{C}$ to remove efficiently adsorbed water. Background spectra were collected on a location next to the sample just before measuring the sample.

FTIR spectra of bulk coals and HCO-tholins samples were collected on $\mathrm{KBr}$ pellets. Pellets were prepared using $300 \mathrm{mg}$ of a $\mathrm{KBr}$ powder previously heated at $300{ }^{\circ} \mathrm{C}$ under vacuum during $3 \mathrm{~h}$. The sample ( $1 \pm 0.1 \mathrm{mg}$ ) was ground and mixed with $300 \mathrm{mg} \mathrm{KBr}$ with a MM200 Retsch grinder equipped with an agate mortar filled with a single ball (30 Hz; 30 minutes). Finally, $13 \mathrm{~mm}$ diameter pellets were prepared using a press Atlas ${ }^{\mathrm{TM}}$ Manuelles (Eurolabo), under 400 bars and the gentle heating at $60{ }^{\circ} \mathrm{C}$ during $1 \mathrm{~h}$ (cooling down during 30 minutes). Infrared spectra were collected under vacuum condition with a Bruker Vertex 70v spectrometer, in the range $4000-400 \mathrm{~cm}^{-1}$ with 200 scans and a $4 \mathrm{~cm}^{-1}$ spectral resolution.

\subsubsection{Solid-state Nuclear Magnetic Resonance}

The $\mathrm{H}^{13} \mathrm{CO}$ - tholins material was characterized by solid NMR using an AVANCE 750 $\mathrm{MHz}$ wide-bore spectrometer $\left(\right.$ Bruker $^{\mathrm{TM}}$ ) operating at MAS rate of $60 \mathrm{kHz}$ designed for $1.3 \mathrm{~mm}$ outside diameter (OD) zirconia rotors (closed with vespel caps). About 1 - $5 \mathrm{mg}$ of materials was put inside the rotor. Under those conditions (188.62 MHz and $60 \mathrm{kHz}$ spinning speed), any rotational sideband was thrown out of the spectral region of interest. This allows a proper deconvolution of spectra. Proton decoupling during acquisition was obtained by using SPINAL64 at a $200 \mathrm{kHz}{ }^{1} \mathrm{H}$ RF field (Fung et al., 2000). All spectra were collected within a $250 \mathrm{kHz}$ spectral windows (1325.3ppm) and 16324 acquisition time domains. To get undistorted lineshapes and filter out background probes signal, the quantitative Direct Polarization (DP) was performed using a speed synchronized spin-echo (Hahn, 1950; Vieira et al., 2016; Lucherelli et al., 2019), pulse sequence $\mathrm{P}_{90} \tau-\mathrm{P}_{180}-\tau$, with the following conditions: $2.75 \mu$ s and $5.5^{\circ} \mathrm{s}$ for $\mathrm{P}_{90^{\circ}}$ and $\mathrm{P}_{180^{\circ}}$ pulses, and echo time being set to $33.6 \mu$ s ( 2 rotation periods). Under such conditions, baseline problems and first order phasing are fixed, which is a prerequisite for quantitative analysis. T1 (spin-lattice relaxation time) were measured following inversion-recovery method (Kingsley, 1999) and led us to set up DP experiments with recycling delays equal to 30s. NMR spectra were processed with the Topspin software (phase and baseline, $\mathrm{LB}=25$ applied). The interpretation of $1 \mathrm{D}{ }^{13} \mathrm{C}$ NMR spectra presents a significant difficulty due to signal overlap. 
Therefore, the spectral decomposition of the spectra was performed with the DMFIT Software developed by Massiot et al. (2002) and previously used for biomass and biochar using Gaussian profiles (Le Brech et al., 2016). The decomposition was repeated three times for the $400{ }^{\circ} \mathrm{C}$ $\mathrm{H}^{13} \mathrm{CO}$ - tholins to estimate the uncertainty on the derived composition due to the processing.

\subsubsection{STXM-XANES analysis}

Synchrotron-based scanning transmission X-ray microscope (STXM) analyses were performed on heated $\mathrm{H}^{12} \mathrm{CO}$ - and $\mathrm{H}^{13} \mathrm{CO}$-tholins to document the carbon speciation using the HERMES STXM beamline at the synchrotron SOLEIL (Saint-Aubin, France) (Belkhou et al., 2015; Swaraj et al., 2017). Samples were prepared either by cryo-ultramicrotomy $\left(\mathrm{H}^{13} \mathrm{CO}-\right.$ tholins) or by depositing the powder on a $\mathrm{Si}_{3} \mathrm{~N}_{4}$ membrane $\left(\mathrm{H}^{12} \mathrm{CO}\right.$-tholin). Energy calibration was done using the well-resolved 3p Rydberg peak of gaseous $\mathrm{CO}_{2}$ at $294.96 \mathrm{eV}$ (Swaraj et al., 2017). XANES hypercube data (stacks) over the carbon absorption range ( $250-350 \mathrm{eV}$ ) were collected with a spatial resolution of $35 \mathrm{~nm}$ at energy increments of $0.1 \mathrm{eV}$ over the $283-291.5$ $\mathrm{eV}$ region, with a dwell time of less than $1 \mathrm{~ms}$ per pixel to prevent irradiation damage (Wang et al., 2009). Stack alignments and extraction of XANES spectra were done using the aXis2000 software (ver2.1n). Normalization of data and quantification of the concentrations of functional groups were done using the QUANTORXS freeware (https://github.com/CorentinLG/QuantORXS/) described in Le Guillou et al. (2018). Note that although this quantification procedure provides consistent results for aromatic, ketonelphenol and carboxyliclester groups, it remains difficult to estimate the concentration of aliphatic groups precisely.

\subsubsection{Raman setup}

Raman measurements were collected with a HORIBA JOBIN-YVON LaRam spectrometer, equipped with a 600 gr. $\mathrm{mm}^{-1}$ groove and operating at $532 \mathrm{~nm}$ (INSU national Facility at ENS-Lyon, France). The laser beam was focused through a $\times 100$ objective. The power onto the sample was $380 \mu \mathrm{W}$, and acquisition time was $5 \times 20$ s. The configuration used yields a lateral resolution of $\approx 1 \mu \mathrm{m}$.

\subsubsection{Analysis of infrared spectra}

For each spectrum, a baseline correction was done using spline functions, and the peak absorbance of the $\mathrm{C}=\mathrm{C}$ band at $1540 \mathrm{~cm}^{-1}$ was set to 1 . The $\mathrm{C}=\mathrm{O}$ and $\mathrm{C}=\mathrm{C}$ bands in $\mathrm{H}^{13} \mathrm{CO}$ tholin spectra at 1656 and $1540 \mathrm{~cm}^{-1}$, respectively, were fitted with Gaussian profiles (using the multi-fit peak functions of the Igor 8 software, Wavemetrics) (Fig.1).

The integrated cross-section is defined by:

$$
\left.A=\int_{v 1}^{v 2} \sigma d v=\int_{v 1}^{v 2} \ln (10) \frac{a(v) \times S}{n} d v \text { (cm/group) (Equation } 1\right)
$$


218 where $a(v)=-\log \left(\frac{I}{I_{0}}\right)$ is the absorbance, with I and $\mathrm{I}_{0}$ are the intensity of the infrared beam 219 after and before the sample, respectively; $n$ is the number of molecular oscillators and $\sigma$ is the 220 absorption cross-section $\left(\mathrm{cm}^{2}\right)$, with $v$ the wavenumber, $v_{1}$ and $v_{2}$ the edges of the spectral range 221 of the spectral component, and $S$ is the probed area $\left(\mathrm{cm}^{2}\right)$.

Finally, the ratio of the absorption cross-section of two chemical groups $X_{1}$ and $X_{2}$ is:

$$
\frac{A_{X 1}}{A_{X 2}}=\frac{a_{X 1}}{a_{X 2}} \times \frac{n_{X 2}}{n_{X 1}} \quad \text { (Equation 2) }
$$

where ax refers to the integrated absorbance of a chemical group $\mathrm{X}$ as aliphatic $\mathrm{CH}_{\mathrm{x}}, \mathrm{C}=\mathrm{O}$ or aromatic species (Aro). acHx was calculated from the integration of the alkyl band between 2750 and $3000 \mathrm{~cm}^{-1}$, while aco and aAro were obtained from a Gaussian fit of the bands at 1656 and $1540 \mathrm{~cm}^{-1}$, respectively. The integrated cross-section ratios of the $\mathrm{CH}_{\mathrm{x}}, \mathrm{C}=\mathrm{O}$ and Aro molecular oscillators, $\mathrm{A}_{C H_{X}} / \mathrm{A}_{A r o}, \mathrm{~A}_{C H_{X}} / \mathrm{A}_{C=O}$ or $\mathrm{A}_{A r o} / \mathrm{A}_{C=O}$ were finally calculated with Equation 2, using estimates from NMR measurements on the $400{ }^{\circ} \mathrm{C} \mathrm{H}^{13} \mathrm{CO}$-tholin. No estimation of individual Ax cross-sections was made, because NMR measurements provided ratios of chemical groups abundances.

Strictly speaking, IR spectroscopy probes normal modes and not bonds. In the case of the $\mathrm{CH}_{\mathrm{x}}$ groups, the mass difference between $\mathrm{H}$ and $\mathrm{C}$ atoms is large and decouples their respective motions. Thus, the corresponding bands are controlled by hydrogen vibration around a single carbon atom. For the terminating carbonyl group, environmental effects from neighboring chemical groups are present, leading to a moderate frequency shift and variations of the absorption coefficient by up to a factor of 2 . But for both $\mathrm{CHx}$ and $\mathrm{C}=\mathrm{O}$, the number of bonds or molecular groups is equal to the number of carbon atoms and a straightforward comparison with ${ }^{13} \mathrm{C}-\mathrm{NMR}$ is possible. Things get harder in the case of aromatic species. A benzene single ring $\left(\mathrm{C}_{6} \mathrm{H}_{6}\right)$, in the gas or as a molecular crystal has a vibration mode at $\sim 1500 \mathrm{~cm}^{-1}$ that involves all carbon atoms (6). Small polycyclic hydrocarbons (1-4 rings) display a sharp narrow band in the range $1700-1500 \mathrm{~cm}^{-1}$, but the number of $\mathrm{C} \cdots \mathrm{C}$ bonds does not equal the number of $\mathrm{C}$ atoms. For instance, naphthalene units have $10 \mathrm{C}$ atoms and $11 \mathrm{C} \cdots \mathrm{C}$ bonds, anthracene and phenanthrene have $14 \mathrm{C}$ atoms and $16 \mathrm{C} \cdots \mathrm{C}$ bonds, and pyrene $16 \mathrm{C}$ atoms and $19 \mathrm{C} \cdots \mathrm{C}$ bonds. In the case of larger polycyclic aromatics, theoretical calculations point to a broad feature peaking around $1600 \mathrm{~cm}^{-1}$, which is due to the E2g normal mode that involves all carbon atoms and phonon propagation (Mapelli et al., 1999). Immature kerogens and coals contain small (14 rings) polycyclic aromatics inserted in a macromolecular network and display a similar broad band at $1600 \mathrm{~cm}^{-1}$. Altogether, the infrared absorption at $1600 \mathrm{~cm}^{-1}$ results from the motion of all carbon atoms, but in contrast no clear connection can be established with a number of $\mathrm{C}=\mathrm{C}$ bonds, nor a number of rings. In this respect, the terms "aromatic ring stretch" or " $\mathrm{C}=\mathrm{C}$ " used in literature are somewhat misleading, and we have used the term "Aro". As such, AAro is controlled by the number of carbon atoms, allowing a direct comparison with ${ }^{13} \mathrm{C}-\mathrm{NMR}$. In the details, we expect significant variations of AAro with the size and local environment of the polyaromatic specie, for instance -OH groups (see discussion below).

Last, the Artificial Bees algorithm was used from the ESA Package PYGMO (https://esa.github.io/pygmo/) and tested two fit models: one based on 7 Gaussian and one on 7 
pseudo-Voigt components. Each group comprised 50 simulations and the fit quality was estimated with a $\chi^{2}$ function. A 20000 bees' population over 50 generations was used. For each of the 200 numerical simulations to get the good fit in term of $\chi^{2}$ value.

\section{RESULTS}

\subsection{Solid-state ${ }^{13} \mathrm{C}$ DP/MAS NMR analysis}

Solid-state ${ }^{13} \mathrm{C}$ DP/MAS NMR spectra of the pristine and heated $\mathrm{H}^{13} \mathrm{CO}$-tholins are presented in Fig. 2a and the band assignment is given in Table 1, following the fitting results in Fig. $2 \mathrm{~b}$ and $1 \mathrm{c}$ for the initial and $400{ }^{\circ} \mathrm{C} \mathrm{H}^{13} \mathrm{CO}$ tholins, respectively. We observe two intense features at 100-160 ppm and $0-100 \mathrm{ppm}$ in the spectra of both samples, which are assigned to $\mathrm{sp}^{2}$ and $\mathrm{sp}^{3}$ carbon atoms, respectively. The range $0-45 \mathrm{ppm}$ usually points to $\mathrm{C}$ atoms in alkyl chains, while the range of 45-90 ppm points to oxygenated aliphatic carbons, e.g. alcoholic, carboxylic or ether groups (Pretsch et al., 2000). It is, however, challenging to separate the respective contribution of these two groups of carbon atoms accurately. $\mathrm{sp}^{2}$ carbons in the 100 160 ppm range can be assigned to olefinic chains (conjugated and unconjugated alkenes) and aromatic or polyaromatic species. We cannot distinguish these two types of speciation. The broad and weak signal which peaks at $170 \mathrm{ppm}$ points to $\mathrm{C}=\mathrm{O}$ groups in esters or carboxylic groups, and that at $210 \mathrm{ppm}$ indicates to $\mathrm{C}=\mathrm{O}$ in ketones or aldehydes. The effect of heating results in a steady increase of unsaturated versus saturated carbons, and a dramatic drop of labile oxygenated groups (Fig. 2a). This is in full agreement with the carbonization process that promotes hydrogen loss, carbonyl loss and aromatization.

The relative abundances $\mathrm{Fx}$, of the different carbon groups, was derived through a decomposition procedure using a fit model based on Gaussian components (Fig. b-c; Table 1) (Massiot et al., 2002, Le Brech et al., 2016). The unheated $\mathrm{H}^{13} \mathrm{CO}$ - tholins appears to be mainly composed of $-\mathrm{CH}_{\mathrm{x}}(\mathrm{x}=1$ to 3$)$ groups $\left(\mathrm{F}_{\mathrm{ali}}=\sim 61 \%\right)$, olefinic carbons ( $\left.\mathrm{F}_{\text {aro }}=\sim 32.5 \%\right)$ and a lower content of $\mathrm{C}=\mathrm{O}$ groups $\left(\mathrm{FCO}_{\mathrm{CO}}=5.1 \%\right)$. The ${ }^{13} \mathrm{C}$ NMR spectrum of the $400{ }^{\circ} \mathrm{C}$ heated $\mathrm{H}^{13} \mathrm{CO}$-tholins is dominated by aromatic carbons $\left(\mathrm{F}_{\mathrm{aro}}=\sim 64 \%\right)$ and contains less aliphatic $-\mathrm{CH}$

\subsection{Raman Analysis}

The Raman spectrum of the unheated $\mathrm{H}^{13} \mathrm{CO}$-tholin is dominated by a strong fluorescence background, with no detectable vibrational bands. The spectra of the heated $\mathrm{H}^{13} \mathrm{CO}$-tholin display the first-order carbon bands superimposed onto a high fluorescence background. The spectral contrast (i.e. the intensity of the $G$ and $D$ bands with respect to the fluorescence intensity) is low, leading to poor quality of the reduced spectra after subtraction of a linear baseline (Fig. S-3). The use of a Lorentzian-Breit-Wigner-Fano fit model was complicated, due to the poor spectral quality at the edge of the $G$ and D bands. Despite these troubles, these data point a G band peak position at $\sim 1537 \mathrm{~cm}^{-1}$ and a FWHM of $\sim 80 \mathrm{~cm}^{-1}$. For comparison with published data, the peak position of a ${ }^{12} \mathrm{C}$-bearing sample can be estimated through $\omega_{12}=\omega_{13}\left(\mu_{12} / \mu_{13}\right)$, with $\omega$ the peak position and $\mu$ the reduced mass. We obtain $\omega_{12}=1593$ $\mathrm{cm}^{-1}$, which points to a nano-graphitic structure in terms of $\mathrm{sp}^{2}$ structure and is entirely consistent 
with a kerogen structure as found in chondritic IOM, coals and terrestrial kerogens (Ferrari \& Robertson, 2001; Quirico et al., 2018). These data support that most of the unsaturated carbon atoms are present in small-sized polyaromatic units, with negligible olefinic carbon bonds.

\subsection{XANES analysis}

STXM-based XANES data revealed that the $\mathrm{H}^{13} \mathrm{CO}$-tholins heated at $400{ }^{\circ} \mathrm{C}$ is homogeneous at the submicrometric level. The prominent feature at $285-285.5 \mathrm{eV}$ of the XANES spectra indicate that more than half of the carbon atoms (56\%) are involved in aromatic/olefinic groups ( $\mathrm{C}=\mathrm{C}$ groups) (Fig. 3a, Table 2). A shoulder is observed at $287.7 \mathrm{eV}$, which can be assigned to the aliphatic carbons $\left(-\mathrm{CH}_{\mathrm{x}}\right)(\sim 30 \%$ of the $\mathrm{C}$ atoms). Additional broad features at $286.2 \mathrm{eV}$ and $288.5 \mathrm{eV}$ are assigned to ketone/phenols $\left(\mathrm{C}=\mathrm{O}\right.$ and $\mathrm{C}_{\mathrm{Ar}}-\mathrm{OH}, \sim 14 \%$ of the $\mathrm{C}$ atoms) and carboxylic/ester (R-COOH,R'; $4 \%$ of the $\mathrm{C}$ atoms). These estimates are consistent with NMR results. The absorption around $286.5 \mathrm{eV}$ could also be assigned to the nitrile functional group, but its presence is discarded by the low elemental N/C ratio (0.01) (Table S-1). Even if our measurements do not report such heterogeneity in $\mathrm{H}^{13} \mathrm{CO}$-tholins, we cannot exclude the presence of such tiny inclusions.

To validate their XANES quantification method, Le Guillou et al. (2018) applied both XANES and DP/MAS NMR techniques to fresh lignin and lignin heated at $300{ }^{\circ} \mathrm{C}$ and $350{ }^{\circ} \mathrm{C}$ given by Le Brech et al. (2016). This work suggested that XANES and NMR quantification methods provide consistent results for aromatic groups, ketonelphenol groups and carboxyliclester groups. However, the aliphatic groups of lignin samples appeared overestimated by about $15 \%$. In the case of the heated $\mathrm{H}^{13} \mathrm{CO}$-tholins, there is a reasonable agreement between XANES and NMR for the aliphatic and the carboxylic groups (Fig. 3b). In contrast, the aromatic groups' abundances determined from XANES are lower than that obtained from DP/MAS NMR, as $55 \pm 4 \%$ and $64 \pm 1 \%$, respectively.

\subsection{Infrared spectroscopy}

The infrared spectra of the initial and $400{ }^{\circ} \mathrm{C} \mathrm{H}^{12} \mathrm{CO}$ and $\mathrm{H}^{13} \mathrm{CO}$-tholins are presented in Fig. 4. The heterogeneity of the $400{ }^{\circ} \mathrm{C} \mathrm{H}^{13} \mathrm{CO}$-tholins was checked in both the $\mathrm{KBr}$ pellet and Micro-FTIR spectra (Supporting Information, Fig. S-4). The broadband at $3600-3100 \mathrm{~cm}^{-1}$ corresponds to the stretching mode of $-\mathrm{OH}$ groups in alcohols, carboxylic groups or phenol ( $\left.\mathrm{Caro}_{\mathrm{O}} \mathrm{OH}\right)$. The peak at $3100-3000 \mathrm{~cm}^{-1}$ is assigned to aromatic C-H. The region of 2800-3000 $\mathrm{cm}^{-1}$ displays four main peaks, including the antisymmetric and symmetric stretching modes of methyl $\left(\mathrm{CH}_{3}\right)$ and methylene $\left(\mathrm{CH}_{2}\right)$ groups and possibly of the $\mathrm{CH}$ group, as a component centered at $\sim 2900 \mathrm{~cm}^{-1}$. However, this spectral range is highly congested and more components are present (see below). The spectral region $1800-1500 \mathrm{~cm}^{-1}$ displays bands at 1656 and 1540 $\mathrm{cm}^{-1}$, and $1708 \mathrm{~cm}^{-1}$ and $1608 \mathrm{~cm}^{-1}$ for the $\mathrm{H}^{13} \mathrm{CO}$ and $\mathrm{H}^{12} \mathrm{CO}$-tholins, respectively. These features (e.g., 1656 and $1540 \mathrm{~cm}^{-1}$ ) are assigned to the stretching modes of the carbonyl $(\mathrm{C}=\mathrm{O})$ and aromatic $(\mathrm{C}=\mathrm{C})$, respectively (Painter et al., 1981). In the region $1500-900 \mathrm{~cm}^{-1}$, bending, deformation and out-of-plane modes of $\mathrm{CH}_{2}, \mathrm{CH}_{3}$ and $\mathrm{C}-\mathrm{O}$ bonds are present at 1450, 1375 and $1300 \mathrm{~cm}^{-1}$. We will follow here Painter et al. (1981) which state that the broad feature peaking 
at $\sim 1200 \mathrm{~cm}^{-1}$ does not provide any significant compositional information, in particular on the abundance of ether groups (-C-O-C-).

\subsubsection{Spectral decomposition of the $\mathrm{CH}_{x}$ stretching region}

The region between $3000-2750 \mathrm{~cm}^{-1}$ results from the contribution of symmetric and antisymmetric stretching modes of $\mathrm{CH}_{2}, \mathrm{CH}_{3}$, possibly of $-\mathrm{CH}$, and contains information on the abundance of alkyl groups and the ratio of $\mathrm{CH}_{2}$ and $\mathrm{CH}_{3}$ groups. However, the spectral congestion makes it difficult to identify the exact number of components, spectral profiles and physical interpretation. Pure alkanes at a low temperature usually display several components ascribed to the $\mathrm{d}^{-}$antisymmetric stretch of chain $\mathrm{CH}_{2}$, the symmetric stretch of terminal $\mathrm{CH}_{2}$ $\left(\mathrm{d}^{+} \omega\right)$ and two components arising from a Fermi resonance between the symmetric stretch of chain $\mathrm{CH}_{2}$ and the overtone of the $\mathrm{H}-\mathrm{C}-\mathrm{H}$ bending mode, reported as $\mathrm{d}^{+}$and $\mathrm{d}^{+} \mathrm{FR}$ (the first being dominating by the fundamental mode) (Fig. 5) (MacPhail et al., 1984); other components are the $\mathrm{r}_{\mathrm{a}}^{-}$and $\mathrm{r}^{-} \mathrm{b}$ in-plane and out-of-plane asymmetric stretch of $-\mathrm{CH}_{3}$, and the symmetric stretch in Fermi resonance with the first overtone of the $\mathrm{CH}_{3}$ bending mode, named $\mathrm{r}^{+}$and $\mathrm{r}^{+}$FR. Pure alkanes measured at low-temperature display peaks and shoulders in agreement with this assignment. However, the different components cannot be guessed for a similar compound in the liquid state at room temperature (Fig. 5). Even more, critical issues arise in the case of natural complex mixtures or solids, such as broader components, spectral shapes changes and peak position shifts. In the case of coals and chondritic IOM, the presence of oxygen as ether or carbonyl groups likely modifies the spectral distribution, making uncertain the spectral decomposition of the bands (Orthous-Daunay et al., 2013; Quirico et al., 2018).

To explore more quantitatively this congestion issue, we ran optimization simulations of the fit of the alkyl band of an IOM sample extracted from the Orgueil (CI1) chondrite (OrthousDaunay et al., 2013). We used the Artificial Bees algorithm from the ESA Package PYGMO (https://esa.github.io/pygmo/) and tested two fit models: one based on 7 Gaussian and one on seven pseudo-Voigt components. Two components aimed to fit the small features at $\sim 3000$ and $2790 \mathrm{~cm}^{-1}$, due to the aromatic $\mathrm{C}-\mathrm{H}$ stretch and a combination mode from fundamental modes in the fingerprint region (Fig. 6). The fit model of the aliphatic bands then consisted of five components, as usually reported in the literature (Dartois et al., 2007, 2004; Orthous-Daunay et al., 2013). We ran two groups of simulations: one keeping constant the Full Width at Half Maximum FWHM of $35 \mathrm{~cm}^{-1}$ for all the components, and another one where FWHM is let free within the range 20-40 $\mathrm{cm}^{-1}$. Each group comprised 50 simulations, and the fit quality was estimated with a $\chi^{2}$ function. A 20000 bees' population over 50 generations were used. For each of the 200 numerical simulations, the resulting fit was excellent in terms of $\chi^{2}$ value (Fig. 6a). The results are summarized in Fig. 6b-c. Two parameters are of particular interest: (1) the ratio of the integrated absorbance of the antisymmetric stretching components of $\mathrm{CH}_{2}$ and $\mathrm{CH}_{3}$ (асн2/асн3) and (2) the sum of these values. The first estimates the ratio of the number of $\mathrm{CH}_{2}$ over $\mathrm{CH}_{3}$ groups, while the second is a measure of the alkyl content ( $\left.\mathrm{acH}_{2}+\mathrm{acH} 3\right)$. Note, however, that for this latter the abundance of the $\mathrm{CH}$ group is not measured regarding the assignment of the central component peaking at $\sim 2900 \mathrm{~cm}^{-1}$ to the overtone of a bending mode in Fermi 
forced to $35 \mathrm{~cm}^{-1}$, we obtain $1.36 \pm 0.07$ and $1.52 \pm 0.28$ for асн2/аснз and $57.0 \pm 1.8$ and 59.0 \pm 2.7 for $\mathrm{acH}_{2}+\mathrm{ach} 3$, for the Gaussian and pseudo-Voigt models, respectively (Fig. 6b). If FWHM is let free within $20-40 \mathrm{~cm}^{-1}$, we obtain $1.37 \pm 0.79$ and $1.66 \pm 0.90$ for асн2/аснз and $50.4 \pm 6.3$ and $52.8 \pm 7$ for асн2 + acH3, for the Gaussian and pseudo-Voigt models, respectively. The dispersion is much larger, and the absolute values of the sum are also significantly different from those obtained by forcing FWHM. To make things clear on the impact in terms of chemical information, we can estimate the $\mathrm{n}_{\mathrm{CH}} / \mathrm{n}_{\mathrm{CH}} 3$ of Orgueil IOM, using the $\mathrm{A}_{\mathrm{CH} 2}$ and $\mathrm{A}_{\mathrm{CH} 3}$ values from Dartois et al. (2007). We find that $\mathrm{n}_{\mathrm{CH} 2} / \mathrm{n}_{\mathrm{CH}}$ ranges between 0.5 and 4.5 , while NMR measurements provide a value ranging 1.9-2.6 (Gardinier et al. 2000; assuming that ether bridges are $\mathrm{CH}_{3}-\mathrm{O}-\mathrm{CH}_{2}: \mathrm{CH}_{2}-\mathrm{O}-\mathrm{CH}_{2}=1: 1$ or $\mathrm{CH}_{2}-\mathrm{O}-\mathrm{CH}_{2}$, respectively. This result is basically of little value. In practice, things may not be as worse, provided that the fit model is similar to that used to derive the absorption cross-sections. Finally, due to the sensitivity of the fit models on derived parameters, and to propose an easy procedure allowing cross-comparison of data collected by independent groups, we prefer to use the integrated absorbance of the whole alkyl band, in place of $\mathbf{a c H}_{2}+\mathbf{a c H}_{3}$. The calibration presented below will lose accuracy when the $\mathrm{n}_{\mathrm{CH} 2} / \mathrm{n}_{\mathrm{CH} 3}$ ratio departs from that of the alkane's standards, but as discussed below, the impact is very limited.

\subsubsection{Determining the ${ }_{C H x} / A_{C=C}$ and $A_{C H x} / A_{C=O}$ ratios}

The ratio of the absorption cross-section of two chemical groups $X_{1}$ and $X_{2}$ is calculated from the Equation 1 . The term $n_{\mathrm{X} 2} / \mathrm{n}_{\mathrm{X} 1}$ is provided by the NMR analysis, and the integrated absorbances of the alkyl, carbonyl and aromatic groups are measured in the spectra of the 400 ${ }^{\circ} \mathrm{C} \mathrm{H}^{13} \mathrm{CO}$-tholins. The uncertainty values were estimated by considering fit uncertainties, the baseline correction, sample heterogeneity (Fig. S-4) and the uncertainty on NMR values of the relative abundance of the three chemical groups. We finally obtain the ratio values of integrated cross-section: $\mathrm{A}_{\mathrm{CHx}} / \mathrm{A}_{\mathrm{Aro}}=1.578 \pm 0.183$ and $\mathrm{A}_{\mathrm{CHx}} / \mathrm{A}_{\mathrm{C}=\mathrm{O}}=0.116 \pm 0.006$ (Table 3).

\section{DISCUSSION}

\subsection{Achievements and limitations}

The composition of the $\mathrm{H}^{13} \mathrm{CO}$-tholin determined by NMR is very reliable for the aliphatic and aromatic contents, due to intense DP/MAS features at 38 and 130 ppm that are unambiguously assigned to alkyl and conjugated carbons. Unlike CP/MAS data, these spectra do not suffer from selective signal enhancement and are suitable for quantification. The assignment is also robust for oxygen-bearing groups, but the features at 169 and $200 \mathrm{ppm}$ are weak and suffer from a higher uncertainty in their relative abundances. The new calibration of $\mathrm{H}^{13} \mathrm{CO}$-tholin allows determining the absorption cross-section ratios of the $\mathrm{CH}_{\mathrm{x}}, \mathrm{C}=\mathrm{O}$ and Aro groups.

Nevertheless, the calibration derived from the $\mathrm{H}^{13} \mathrm{CO}$-tholins suffers from limitations and biases that are intrinsic to infrared spectroscopy. First, as we estimate the alkyl abundance through the integration of all the alkyl bands $\left(\mathrm{CH}_{\mathrm{x}}\right.$ stretching in the range of $\left.3000-2800 \mathrm{~cm}^{-1}\right)$, 
the validity of the $\mathrm{H}^{13} \mathrm{CO}$-tholins as a calibration standard relies on a similar distribution of the alkyl groups in the kerogen and coal samples that are analyzed. Hence, the absorption crosssection of $\mathrm{CH}_{2}$ and $\mathrm{CH}_{3}$ are not identical, e.g. for the anti-symmetric stretching modes Асн3 = $(11.8 \pm 0.76) \times 10^{-18}$ and $\mathrm{AcH}_{2}=(7.7 \pm 1.5) \times 10^{-18} \mathrm{~cm} /$ group (Dartois et al., 2007; Sandford et al., 1991). Considering a variation range of $1.5-3.0$ for $\mathrm{n}_{\mathrm{CH} 2} / \mathrm{n}_{\mathrm{CH} 3}$ that is observed in chondritic IOM (Orthous-Daunay et al., 2013), we can calculate the variation of the sum of the integrated absorbance of the anti-symmetric stretching modes of $\mathrm{CH}_{2}$ and $\mathrm{CH}_{3}, \mathrm{a}=\mathrm{acH} 2+\mathrm{acH}$, as:

$$
\frac{a_{1.5}}{a_{3}}=\frac{1+\frac{1}{1.5} \frac{A_{C H 3}}{A_{C H 2}}}{1+\frac{1}{3} \frac{A_{C H 3}}{A_{C H 2}}}=1.18
$$

This means that $\mathrm{n}_{\mathrm{CH} 2} / \mathrm{ncH}_{\mathrm{CH}}$ estimates on IOM from thermally processed chondrites obtained with our calibration are underestimated by $20 \%$ at maximum. Second, a critical issue with the $1600 \mathrm{~cm}^{-1}$ band is the sensitivity of its absorption cross-section to the oxygen molecular environment, in particular the phenolic $(-\mathrm{OH})$ and possibly the ether (C-O-C) groups (Painter et al., 1981). This means that the kerogen to be analyzed should be as similar as possible to the calibration standard in terms of oxygen speciation around aromatic carbons. In this respect, the $400^{\circ} \mathrm{C} \mathrm{H}^{13} \mathrm{CO}$-tholins do not fit mature samples that have large polyaromatic units, and which are known to have a lower aromatic cross-section. This issue is probably more critical for coals, due to the large $\mathrm{O} / \mathrm{C}$ variations upon maturity.

\subsection{IOM composition of primitive chondrites}

A way to test our calibration is to apply our method to samples whose composition has been previously determined by NMR. We report here the composition ( $\mathrm{n}_{\mathrm{CH}} / \mathrm{n}_{\mathrm{C}=\mathrm{O}}$ and $\mathrm{n}_{\mathrm{CHx}} / \mathrm{n}_{\mathrm{C}=\mathrm{C}}$ ) of the IOMs from the Orgueil, Murchison, EET 92042 and Tagish Lake chondrites, derived from the spectra published by Orthous-Daunay et al. (2013) (Fig. S-5). These compositions are compared with the NMR estimates of Cody \& Alexander, (2005) and Gardinier et al. (2000) (Fig. 7). It is first necessary to discuss those NMR data, which display some inconsistencies between them.

Two types of NMR measurements are presented in these publications: Single-pulse (SP) MAS and Cross Polarized (CP) MAS. Single-pulse (SP) MAS NMR is usually presented as suitable for quantification (Baccile et al., 2014), but in the case of natural samples $\left({ }^{13} \mathrm{C} /{ }^{12} \mathrm{C} \sim\right.$ $1 \%$ ), the signal is low, long acquisition time is required, and eventually the spectra still contain significant noise. In the case of broad features, band distortion can happen due to dephasing processes before spectrum acquisition, leading to significant errors in the quantification of band integral. In addition, the baseline may depart from an ideal flat line, thereby perturbing the profile of broad features. Altogether, these artifacts may lead to significant errors. These issues can be fixed, at least partly, by using spin echo DP/MAS experimental configuration, as done here on the standard samples. By applying two subsequent pulses ( $\mathrm{P}_{90}-\tau-\mathrm{P}_{180}-\tau$, with $\tau$ being a multiple of the MAS frequency), a rephasing of the signal is obtained, and broad features are not anymore distorted (Hahn, 1950; Vieira et al., 2016; Lucherelli et al., 2019). Cross polarization Magic Angle Spinning (CP/MAS) analysis is based on the magnetization transfer 
from hydrogen to carbon atoms. It provides spectra with an improved signal-to-noise ratio, but the quantification is not straightforward (Baccile et al., 2014; dela Rosa et al., 1992). Thus, the intensity of the signal arising from a carbon atom depends on its hydrogen environment, and the signal depends on the contact time. It requires an estimate of the fraction of undetected carbon atoms through calculations constrained by the elemental composition of the sample. The CP/MAS estimation is then much less direct than DP/MAS, and may suffer from error propagation on speciation quantitation and elemental composition, as well as a noncomprehensive identification of all involved carbon speciation.

Cody and Alexander, (2005) and Gardinier et al. (2000) both report CP/MAS NMR data that provide quantitative estimates of the composition of IOM from Orgueil and Murchison. In the case of the Murchison chondrite, the ratio of aliphatic to aromatic carbon atoms $\mathrm{F}_{\text {ali }} / \mathrm{F}_{\text {aro }}$ estimated by Gardinier et al. (2000) is slightly higher than that of Cody and Alexander (2005) but it lies within the error bars (Fig. 8). In the case of Orgueil, the Fali/Faro ratio from is lower than that of Cody and Alexander (2005), but the error bars also overlap (Fig. 8). In contrast, there is some significant disagreement regarding carbon atoms in oxygenated functions, in particular for Orgueil (Fig. 8). These differences may be due to different experimental procedures. Gardinier et al. (2000) investigated the spectral dependence upon contact time, applied a correction accounting for undetected carbon atoms based on the bulk H/C ratio, determined from the combustion experiment. The abundance of undetected aromatic carbon was found significant, ranging from $20-29 \%$. We note that the $\mathrm{H} / \mathrm{C}$ value $(0.7$ in their study is higher than that measured by Alexander et al. (2007, 2013) (0.588 \pm 0.016 and 0.618 , respectively), which may have an impact on the derived abundances. Cody and Alexander (2005) did not run systematic measurements as a function of contact time, but this latter was set to $4.5 \mathrm{~ms}$ to fit with SP/MAS spectra. This quantitative interpretation of CP/MAS spectra without investigations at variable contact time is not usual, and estimates might suffer from biases (S. Derenne, personal communication; see also). In fact, in the details, the estimates derived by Cody and Alexander (2005) from SP/MAS and CP/MAS show significant differences. Due to these complex issues with CP/MAS, we have restricted to the SP/MAS data from Cody and Aleander (2005) for comparison with our FTIR data.

We observe that $\mathrm{n}_{\mathrm{CH}} / \mathrm{n}_{\mathrm{C}=\mathrm{C}}$ estimated from infrared data following equation 2 fits well NMR data in the case of Orgueil, Murchison and Tagish Lake. $\mathrm{n}_{\mathrm{CH}} / \mathrm{n}_{\mathrm{C}=\mathrm{C}}$ in EET 92042 is underestimated by a factor of $\sim 1.3$, though the error bars overlap (Fig. 8). The first explanation of this moderate discrepancy is the chemical control of the AAro absorption cross-section by oxygenated groups (Painter et al., 1981). The O/C ratio in EET $92042(\mathrm{O} / \mathrm{C}=16.6 \pm 0.2)$ is lower than those of Murchison (18.3 \pm 0.3$)$ and Orgueil $(18.1 \pm 0.2)$. These values are quite similar. According to NMR data, the main oxygen speciation in chondritic IOM is carbonyl (ketone, ester) and ether bridges. The estimations from SP/MAS NMR spectra are challenging due to the faintness of the features and the poor signal-to-noise ratio, and they do not point to significant variations between EET 92042, and Orgueil and Murchison. Altogether, there is no evidence of a chemical control of the AAro absorption cross-section. A second tentative explanation is that the SP/MAS data are not quantitative enough, due to the biases abovementioned. The only way to firmly solve this issue would be to collect DP/MAS NMR spectra (MAS synchronized spin echoes) of IOMs from those three chondrites. 
Regarding carbonylated species, we observe that $\mathrm{n}_{\mathrm{CH}} / \mathrm{n}_{\mathrm{C}=\mathrm{O}}$ is systematically higher than NMR estimates, though the hierarchy Orgueil > Murchison $>$ Tagish Lake is similar to NMR estimates (Fig. 8). The number of bonds is equal to the number of carbon atoms in the case of $\mathrm{CH}_{\mathrm{x}}$ groups and carbonyl; therefore, the difference may be due here to different absorption cross-sections of the $\mathrm{C}=\mathrm{O}$ group between these IOMs and the HCO-tholin standard. As for the $\mathrm{C}=\mathrm{C}$ bonds, the local molecular environment plays a role in the control of the absorption crosssection of the $\mathrm{C}=\mathrm{O}$ stretching mode. Such variations have been observed for small molecules in the solid-state, for instance, amorphous ices of formic acid and acetaldehyde, whose carbonyl absorption cross-sections differ by a factor of 2 (Bouilloud et al., 2015; Hudson \& Ferrante, 2020). Nevertheless, as mentioned in the previous section, the re-analysis of these IOM samples by DP/MAS NMR (MAS synchronized spin echoes) is required to get a firm conclusion.

To sum up, the $\mathrm{H}^{13} \mathrm{CO}$-tholins standard provides a suitable calibration for estimating $n_{C H x} / n_{C=C}$ in IOMs derived from FTIR measurement compared with SP/MAS NMR. In contrast, $n_{C H x} / n_{C=O}$ is overestimated in all classes of chondrites compared with the SP/MAS NMR from Cody and Aleander (2005).

\subsection{Composition of ligno-cellulosic coals}

We report now the composition of a series of coals of varying ranks from lignite to semianthracite (Table S-2, Fig. S-5). The majority of coals plot within the Type III kerogen band on the "Van Krevelen" diagram (Fig. 9a). Due to spectral congestion, the fit in the region 1800$1500 \mathrm{~cm}^{-1}$ region is more complicated than for IOMs. We used four spectral components due to ketones /quinone, aromatic branched with phenolic -OH (or -O-), and aromatic with a carboxylic group (Chen et al., 2012; Painter et al., 1981; Petersen et al., 2008) (Fig. S-6). The abundance of aromatic groups is then estimated through the two last components, and the $\mathrm{C}=\mathrm{O}$ by the first two components. Our results of the evolution trends are consistent with previous estimations based on integrated abundance derived from IR spectra (Chen et al., 2012). No NMR data are available for our coals, but NMR data are available for Chinese series of coals (Wei \& Tang, 2018) and for the so-called Argonne Premium coals, a subset of the Penn State Coals Data bank (dela Rosa et al., 1992). Figure 9b-c reports the FTIR and NMR CP/MAS estimates of Wei and Tang (2018) plotted against the vitrinite reflectance. We observe two opposite trends: while the NMR estimates point to a progressive decrease of $\mathrm{n}_{\mathrm{CHx}} / \mathrm{n}_{\mathrm{C}=\mathrm{C}}$, the FTIR estimates show an increase up to the vitrinite reflectance of $1.73 \%$ and then a decline for the semi-anthracite samples. In contrast, $\mathrm{n}_{\mathrm{CO}} / \mathrm{n}_{\mathrm{CHx}}$ is consistent with NMR. This situation is opposite to what he had with IOMs (Fig. 8).

The NMR estimates of Wei and Tang (2018) are, at least qualitatively, in agreement with the progressive aromatization of the coals during diagenesis and catagenesis (Chen et al., 2012). This supports the view that the ratio of the cross-section $A_{\mathrm{CHx}} / A_{\text {Aro }}$ varies with the vitrinite reflectance, as previously reported (Painter et al., 1981; Dela Rosa et al., 1992). It also shows that any semi-quantitative analysis based on IR measurements leads to inaccurate estimates of the chemical evolution. NMR data collected by Wei \& Tang (2018) were obtained in CP/MAS with a 3 ms contact time. The publication does not report measurements over variable time 
contact, nor the acquisition of single pulse spectra. In contrast, Dela Rosa et al. (1992) report varying contact time CP/MAS measurements on Argonne Premium coals and one single pulse (Bloch decay) analysis of Pittsurgh $n^{\circ} 8$ that fits the corresponding CP/MAS estimate. Muntean et al. (1990) also report consistent CP/MAS and single pulse estimates in the case of Pocahontas $\mathrm{N}^{\circ}$ 3, provided that the delay time remains $<75 \mu \mathrm{s}$. However, the agreement between both types of measurements may be worse in the case of O-rich low-rank coals (Dela Rosa et al., 1992). We have recalculated the aromaticity of the coals from Wei and Tang (2018) following the definition of Dela Rosa et al. (1992), and found that the difference between estimates of the two sets of data does not exceed $5 \%$.

Finally, using those data and keeping in mind that uncertainties might be worse for the more immature coals, we have calculated the cross-section ratio $\mathrm{A}_{\mathrm{CH}} / \mathrm{Ac}_{\mathrm{C}=\mathrm{C}}$ upon the vitrinite reflectance (Fig. 9d). This curve is best fitted with a $6^{\text {th }}$ order polynomial (Equation 3):

$$
\frac{A_{C H x}}{A_{C=C}}=\sum_{i=0}^{6} K_{i}\left(x-x_{0}\right)^{i}
$$

\section{(Equation 3)}

with $\mathrm{K}_{0}=0.64599 ; \mathrm{K}_{1}=0.23482 ; \mathrm{K}_{2}=6.3733 ; \mathrm{K}_{3}=-16.939 ; \mathrm{K}_{4}=23.446 ; \mathrm{K}_{5}=-14.846$; $\mathrm{K}_{6}=3.7045$ and $\mathrm{X}_{0}=0.3$. This formula applies in the range $\mathrm{R}_{0}=0.3-2 \%$, provided that a similar fit model is used to decompose the coal infrared spectra. We observe that $\mathrm{AcHx}_{\mathrm{C}} / \mathrm{Ac}_{\mathrm{C}=\mathrm{C}}$ increases and $A_{C=C}$ decreases, upon increasing the vitrinite reflectance $R_{0}$, consistently with control of the absorption cross-section by oxygenated groups. The increase is quite high, around one order of magnitude, which means that semi-quantitative sets of infrared data must be imperatively corrected. However, further investigations combining DP/MAS NMR and FTIR measurements on the same series of coals should provide more accurate data.

\section{CONCLUSION}

We have tested a protocol for quantifying the abundance of the $\mathrm{CHx}, \mathrm{C}=\mathrm{O}$ and $\mathrm{C}=\mathrm{C}$ groups in chondritic IOMs and coals, based on a ${ }^{13} \mathrm{C}$ model material characterized by DP/MAS NMR and FTIR. The ratios of the cross-sections of these groups have been determined, and the method has been applied to IOM samples extracted from CI, CM and CR chondrites, and to a series of coals. The estimate of $\mathrm{n}_{\mathrm{CHx}} / \mathrm{n}_{\mathrm{C}=\mathrm{C}}$ in IOM from CI and $\mathrm{CM}$ chondrites is fair, but $\mathrm{nCHx}_{\mathrm{x}} / \mathrm{nC}_{\mathrm{C}} \mathrm{O}$ is overestimated. The estimate of $\mathrm{nCHx}_{\mathrm{n}} / \mathrm{n}_{\mathrm{C}} \mathrm{C}$ in the $\mathrm{CR}$ chondrite is lower than the estimation by SP/MAS published earlier. These discrepancies are due to the control of the absorption of the $\mathrm{C}=\mathrm{O}$ and $\mathrm{C}=\mathrm{C}$ bonds by the local oxygenated chemical structure. In this regard, the oxygen speciation in CR chondrites may be significantly different than that of CI and CM chondrites. However, new NMR analyses of IOM from CR chondrites are necessary to test the reproducibility of existing data. Regarding coals, the use of published NMR analyses shows that the ratio $\mathrm{A}_{\mathrm{CHx}} / \mathrm{Ac}_{\mathrm{C}=\mathrm{C}}$ varies with the vitrinite reflectance over an order of magnitude. Here as well, the local oxygen speciation plays a critical control on AAro, which decreases with increasing the vitrinite reflectance. We propose an analytical law for $A_{\mathrm{CHx}} / \mathrm{A}_{\mathrm{Aro}}$, which should be helpful to determine the aromaticity of any coal sample, provided its vitrinite reflectance is known. 


\section{ACKNOWLEDGEMENTS}

586

587

588

589

590

591

592

593

594

595

596

597

598

599

600

601

602

603

604

605

606

607

608

609

610

611

612

613

614

615

616

617

618

619

620

621

622

623

624

625

626

627

The authors are grateful to the financial support of the H2020 European Research Council (ERC) (SOLARYS ERC-CoG2017_771691). We acknowledge Dr. Julien Alleon for the assistance during STXM-XANES measurements at the HERMES beamline at the SOLEIL Synchrotron.

We also acknowledge Anne-Marie Blanchenet who prepared the microtomed section for STXM analysis at the microscopy platform of the Lille University. We are thankful to the Raman facility in ENS-Lyon, supported by the Institut National des Sciences de l'Univers (INSU). We also thank Paul Craddock and an anonymous reviewer for helpful comments that improved the manuscript.

\section{Supplementary data}

Supplementary data related to this article can be found at the Supporting Information part.

\section{Reference}

Abarghani, A., Gentzis, T., Shokouhimehr, M., Liu, B., \& Ostadhassan, M. (2020). Chemical heterogeneity of organic matter at nanoscale by AFM-based IR spectroscopy. Fuel, 261(116454). https://doi.org/10.1016/j.fuel.2019.116454

Alcouffe, G., Cavarroc, M., Cernogora, G., Ouni, F., Jolly, A., Boufendi, L., \& Szopa, C. (2010). Capacitively coupled plasma used to simulate Titan's atmospheric chemistry. Plasma Sources Science and Technology, 19(1). https://doi.org/10.1088/09630252/19/1/015008

Baccile, N., Falco, C., \& Titirici, M. M. (2014). Characterization of biomass and its derived char using 13C-solid state nuclear magnetic resonance. Green Chemistry, 16(12), 48394869. https://doi.org/10.1039/c3gc42570c

Battandier, M., Bonal, L., Quirico, E., Beck, P., Engrand, C., Duprat, J., \& Dartois, E. (2018). Characterization of the organic matter and hydration state of Antarctic micrometeorites: A reservoir distinct from carbonaceous chondrites. Icarus, 306, 74-93. https://doi.org/10.1016/j.icarus.2018.02.002

Beck, P., Quirico, E., Montes-hernandez, G., L., B., J., B., Orthous-Daunay, F.-R., ... O., B. (2010). Hydrous mineralogy of CM and CI chondrites from infrared spectroscopy and their relationship with low Albedo asteroid. In 41st Lunar and Planetary Science.

Belkhou, R., Stanescu, S., Swaraj, S., Besson, A., Ledoux, M., Hajlaoui, M., \& Dalle, D. (2015). HERMES: A soft X-ray beamline dedicated to X-ray microscopy. Journal of Synchrotron Radiation, 22, 968-979. https://doi.org/10.1107/S1600577515007778

Bouilloud, M., Fray, N., Bénilan, Y., Cottin, H., Gazeau, M. C., \& Jolly, A. (2015). Bibliographic review and new measurements of the infrared band strengths of pure molecules at $25 \mathrm{~K}$ : H2O, CO2, CO, CH4, NH3, $\mathrm{CH} 3 \mathrm{OH}, \mathrm{HCOOH}$ and $\mathrm{H} 2 \mathrm{CO}$. Monthly Notices of the Royal Astronomical Society, 451(2), 2145-2160. https://doi.org/10.1093/mnras/stv1021

Chen, Y., Mastalerz, M., \& Schimmelmann, A. (2012). Characterization of chemical functional groups in macerals across different coal ranks via micro-FTIR spectroscopy. International Journal of Coal Geology, 104, 22-33. https://doi.org/10.1016/j.coal.2012.09.001 
Cody, G. D., \& Alexander, C. M. O. D. (2005). NMR studies of chemical structural variation of insoluble organic matter from different carbonaceous chondrite groups. Geochimica et Cosmochimica Acta, 69(4), 1085-1097. https://doi.org/10.1016/j.gca.2004.08.031

Craddock, P. R., Doan, T. V. Le, Bake, K., Polyakov, M., Charsky, A. M., \& Pomerantz, A. E. (2015). Evolution of Kerogen and Bitumen during Thermal Maturation via SemiOpen Pyrolysis Investigated by Infrared Spectroscopy. Energy and Fuels, 29, 21972210. https://doi.org/10.1021/ef5027532

Dartois, E., Geballe, T. R., Pino, T., Cao, A.-T., Jones, A., Deboffle, D., ... D’Hendecourt, L. (2007). IRAS 08572+3915: Constraining the aromatic versus aliphatic content of interstellar HACs. Astronomy \& Astrophysics, 463, 635-640. https://doi.org/10.1051/0004-6361

Dartois, E., Munoz Caro, G. M., Deboffle, D., \& D’Hendecourt, L. (2004). Diffuse interstellar medium organic polymers Photoproduction of the 3.4, 6.85 and $7.25 \mu \mathrm{m}$ features. Astronomy \& Astrophysics, 423, 33-36.

Dela Rosa, L., Pruski, M., Lang, D., Gerstein, B., \& Solomon, P. (1992). Characterization of the Argonne Premium Coals by using $1 \mathrm{H}$ and 13C NMR and FT-IR spectroscopies. Energy and Fuels, 6(4), 460-468. https://doi.org/10.1021/ef00034a016

Ferrari, A. C., \& Robertson, J. (2001). Resonant Raman spectroscopy of disordered, amorphous, and diamondlike carbon. Physical Review B - Condensed Matter and Materials Physics, 64(7), 1-13. https://doi.org/10.1103/PhysRevB.64.075414

Fung, B. M., Khitrin, A. K., \& Ermolaev, K. (2000). An Improved Broadband Decoupling Sequence for Liquid Crystals and Solids. Journal of Magnetic Resonance, 142(1), 97101. https://doi.org/10.1006/jmre.1999.1896

Gardinier, a, Derenne, S., Robert, F., Behar, F., Largeau, C., \& Maquet, J. (2000). Solid state CP/MAS 13C NMR of the insoluble organic matter of the Orgueil and Murchison meteorites: quantitative study. Earth and Planetary Science Letters, 184, 9-21. https://doi.org/http://dx.doi.org/10.1016/S0012-821X(00)00300-9

Guillou, C. Le, Bernard, S., Pena, F. De, \& Brech, Y. Le. (2018). XANES-Based Quantification of Carbon Functional Group Concentrations. Analytical Chemistry, 90, 8379-8386. research-article. https://doi.org/10.1021/acs.analchem.8b00689

Hahn, E. L. (1950). Spin Echoes. Phys Rev Lett.

Hudson, R. L., \& Ferrante, R. F. (2020). Quantifying acetaldehyde in astronomical ices and laboratory analogues: IR spectra, intensities, 13C shifts, and radiation chemistry. Monthly Notices of the Royal Astronomical Society, 492, 283-293. https://doi.org/10.1093/mnras/stz3323

Jakob, D. S., Wang, L., Wang, H., \& Xu, X. G. (2019). Spectro-Mechanical Characterizations of Kerogen Heterogeneity and Mechanical Properties of Source Rocks at $6 \mathrm{~nm}$ Spatial Resolution. Analytical Chemistry, 91(14), 8883-8890. https://doi.org/10.1021/acs.analchem.9b00264

Jubb, A. M., Birdwell, J. E., Hackley, P. C., Hatcherian, J. J., \& Qu, J. (2020). Nanoscale Molecular Composition of Solid Bitumen from the Eagle Ford Group across a Natural Thermal Maturity Gradient. Energy and Fuels, 34(7), 8167-8177. https://doi.org/10.1021/acs.energyfuels.0c00963

Kebukawa, Y., Alexander, C. M. O. D., \& Cody, G. D. (2011). Compositional diversity in insoluble organic matter in type 1, 2 and 3 chondrites as detected by infrared spectroscopy. Geochimica et Cosmochimica Acta, 75(12), 3530-3541. 
Kebukawa, Y., Kobayashi, H., Urayama, N., Baden, N., \& Kondo, M. (2018). Nanoscale infrared imaging analysis of carbonaceous chondrites to understand organic-mineral interactions during aqueous alteration. PNAS, 1-6. https://doi.org/10.1073/pnas.1816265116

Kingsley, P. B. (1999). Methods of measuring spin-lattice (T1) relaxation times: An annotated bibliography. Concepts in Magnetic Resonance, 11(4), 243-276. https://doi.org/10.1002/(SICI)1099-0534(1999)11:4<243::AID-CMR5>3.0.CO;2-C

Le Brech, Y., Raya, J., Delmotte, L., Brosse, N., Gadiou, R., \& Dufour, A. (2016). Characterization of biomass char formation investigated by advanced solid state NMR. Carbon, 108, 165-177. https://doi.org/10.1016/j.jrp.2009.07.007

Le Guillou, C., Bernard, S., De La Pena, F., \& Le Brech, Y. (2018). XANES-Based Quantification of Carbon Functional Group Concentrations. Analytical Chemistry, 90(14), 8379-8386. https://doi.org/10.1021/acs.analchem.8b00689

Li, W., Zhu, Y. M., Wang, G., \& Jiang, B. (2016). Characterization of coalification jumps during high rank coal chemical structure evolution. Fuel, 185, 298-304. https://doi.org/10.1016/j.fuel.2016.07.121

Lis, G. P., Mastalerz, M., Schimmelmann, A., Lewan, M. D., \& Stankiewicz, B. A. (2005). FTIR absorption indices for thermal maturity in comparison with vitrinite reflectance R0 in type-II kerogens from Devonian black shales. Organic Geochemistry, 36(11), 15331552. https://doi.org/10.1016/j.orggeochem.2005.07.001

Lucherelli, M. A., Raya, J., Edelthalhammer, K. F., Hauke, F., Hirsch, A., Abellán, G., \& Bianco, A. (2019). A Straightforward Approach to Multifunctional Graphene. Chemistry - A European Journal, 25(57), 13218-13223. https://doi.org/10.1002/chem.201903165

MacPhail, R. A., Strauss, H. L., Snyder, R. G., \& Eiliger, C. A. (1984). C-H stretching modes and the structure of n-alkyl chains. 2. Long, all-trans chains. Journal of Physical Chemistry, 88(3), 334-341. https://doi.org/10.1021/j150647a002

Mapelli, C., Castiglioni, C., Zerbi, G., \& Mullen, K. (1999). Common force field for graphite and polycyclic aromatic hydrocarbons. Physical Review B - Condensed Matter and Materials Physics, 60(18), 12710-12725. https://doi.org/10.1103/PhysRevB.60.12710

Massiot, D., Fayon, F., Capron, M., King, I., Le Calvé, S., Alonso, B., ... Hoatson, G. (2002). Modelling one- and two-dimensional solid-state NMR spectra. Magnetic Resonance in Chemistry, 40(1), 70-76. https://doi.org/10.1002/mrc.984

Mathurin, J., Dartois, E., Pino, T., Engrand, C., Duprat, J., Deniset-besseau, A., ... Dazzi, A. (2019). Nanometre-scale infrared chemical imaging of organic matter in ultracarbonaceous Antarctic micrometeorites ( UCAMMs ). Astronomy \& Astrophysics, 160, $1-9$.

Orthous-Daunay, F. R., Quirico, E., Beck, P., Brissaud, O., Dartois, E., Pino, T., \& Schmitt, B. (2013). Mid-infrared study of the molecular structure variability of insoluble organic matter from primitive chondrites. Icarus, 223, 534-543. https://doi.org/10.1016/j.icarus.2013.01.003

Painter, P. P., Snyder, R. W., Starsinic, M., Coleman, M. M., Kuehn, D. W., \& Davis, A. (1981). Concerning the application of FT-IR to the study of coal: A critical assessment of band assignements and the application of spectral analysis programs. Applied Spectroscopy, 35(5), 475-485. https://doi.org/10.1366/0003702814732256

Petersen, H. I., Rosenberg, P., \& Nytoft, H. P. (2008). Oxygen groups in coals and alginite- 
rich kerogen revisited. Coal Geology, 74, 93-113. https://doi.org/10.1016/j.coal.2007.11.007

Pretsch, E., Bühlmann, P., \& Affolter, C. (2000). Structure Determination of Organic Compounds. Structure Determination of Organic Compounds. Springer. https://doi.org/10.1007/978-3-662-04201-4

Quirico, E, Bonal, L., Beck, P., Alexander, C. M. O. D., Yabuta, H., Nakamura, T., ... Schmitt-kopplin, P. (2018). Prevalence and nature of heating processes in CM and C2ungrouped chondrites as revealed by insoluble organic matter. Geochimica et Cosmochimica Acta, 241, 17-37. https://doi.org/10.1016/j.gca.2018.08.029

Quirico, Eric, Orthous-Daunay, F. R., Beck, P., Bonal, L., Brunetto, R., Dartois, E., ... Duprat, J. (2014). Origin of insoluble organic matter in type 1 and 2 chondrites: New clues, new questions. Geochimica et Cosmochimica Acta, 136, 80-99. https://doi.org/10.1016/j.gca.2014.03.025

Russell, L. M., Takahama, S., Liu, S., Hawkins, L. N., Covert, D. S., Quinn, P. K., \& Bates, T. S. (2009). Oxygenated fraction and mass of organic aerosol from direct emission and atmospheric processing measured on the R/V Ronald Brown during TEXAQS/GoMACCS 2006. Journal of Geophysical Research Atmospheres, 114(7), 115. https://doi.org/10.1029/2008JD011275

Sandford, S. A., Allamandola, L. J., Tielens, A. G. G. M., Sellgren, K., Tapia, M., \& Pendleton, Y. (1991). The interstellar C-H stretching band near 3.4 microns: constraints on the composition of organic material in the diffuse interstellar medium. The Astrophysical Journal, 371, 607-620.

Sciamma-O’Brien, E., Carrasco, N., Szopa, C., Buch, A., \& Cernogora, G. (2010). Titan’s atmosphere: An optimal gas mixture for aerosol production? Icarus, 209(2), 704-714. https://doi.org/10.1016/j.icarus.2010.04.009

Sun, X. (2005). The investigation of chemical structure of coal macerals via transmitted-light FT-IR microspectroscopy. Spectrochimica Acta - Part A: Molecular and Biomolecular Spectroscopy, 62, 557-564. https://doi.org/10.1016/j.saa.2005.01.020

Swaraj, S., Belkhou, R., Stanescu, S., Rioult, M., Besson, A., \& Hitchcock, A. P. (2017). Performance of the HERMES beamline at the carbon K-edge. Journal of Physics: Conference Series, 849(1). https://doi.org/10.1088/1742-6596/849/1/012046

Szopa, C., Cernogora, G., Boufendi, L., Correia, J. J., \& Coll, P. (2006). PAMPRE : A dusty plasma experiment for Titan's tholins production and study. Planetary and Space Science, 54, 394-404. https://doi.org/10.1016/j.pss.2005.12.012

Takahama, S., Johnson, A., Russell, L. M., Takahama, S., \& Johnson, A. (2013). Quantification of Carboxylic and Carbonyl Functional Groups in Organic Aerosol Infrared Absorbance Spectra. Aerosol Science and Technology, 47(3), 310-325. https://doi.org/10.1080/02786826.2012.752065

Vieira, M. A., Gonçalves, G. R., Cipriano, D. F., Schettino, M. A., Silva Filho, E. A., Cunha, A. G., ... Freitas, J. C. C. (2016). Synthesis of graphite oxide from milled graphite studied by solid-state 13C nuclear magnetic resonance. Carbon, 98, 496-503. https://doi.org/10.1016/j.carbon.2015.11.037

Wang, J., Morin, C., Li, L., Hitchcock, A. P., Scholl, A., \& Doran, A. (2009). Radiation damage in soft X-ray microscopy. Journal of Electron Spectroscopy and Related Phenomena, 170(1-3), 25-36. https://doi.org/10.1016/j.elspec.2008.01.002

Wei, Q., \& Tang, Y. (2018). 13C-NMR study on structure evolution characteristics of high- 
organic-sulfur coals from typical chinese areas. Minerals, 8(2). https://doi.org/10.3390/min8020049

Xueqiu, H., Xianfeng, L., Baisheng, N., \& Dazhao, S. (2017). FTIR and Raman spectroscopy characterization of functional groups in various rank coals. Fuel, 206, 555-563. https://doi.org/10.1016/j.fuel.2017.05.101

Yabuta, H., Naraoka, H., Sakanishi, K., \& Kawashima, H. (2005). Solid-state 13C NMR characterization of insoluble organic matter from Antarctic CM2 chondrites: Evaluation of the meteoritic alteration level. Meteoritics and Planetary Science, 40(5), 779-787. https://doi.org/10.1111/j.1945-5100.2005.tb00979.x

Yang, J., Hatcherian, J., Hackley, P. C., \& Pomerantz, A. E. (2017). Nanoscale geochemical and geomechanical characterization of organic matter in shale. Nature Communications, 8(2179). https://doi.org/10.1038/s41467-017-02254-0 
780 Table 1. Chemical shifts, corrected relative abundances of the different functional carbon 781 groups in the initial and heated $\mathrm{H}^{13} \mathrm{CO}$-tholins derived from Figure $1 \mathrm{~b}-\mathrm{c}$.

782 Table 2. The functional carbon groups concentrations given by the XANES-based 783 quantification (Le Guillou et al., 2018).

784 Table 3. Integrated cross-section ratio calculation of the $400{ }^{\circ} \mathrm{C} \mathrm{H}^{13} \mathrm{CO}$-tholins based on the 785 quantitative NMR and the infrared spectral decomposition. 
Figure 1. (a) The unheated and heated at $400{ }^{\circ} \mathrm{C} \mathrm{H}^{13} \mathrm{CO}$-Tholins recorded with a sample

788

789

790

791

792

793

794

795

796

797

798

799

800

801

802

803

804

805

806

807

808

809

810

811

812

813

814

815

816

817

818

819

820

821

822

823

spinning rate of $60 \mathrm{kHz}$ and interscan delay of 30 seconds in the comparison with the IOM Murchison and Orgueil (Cody et al., 2005, 2002, Gardinier et al., 2000). Deconvolution of the solid state ${ }^{13} \mathrm{C}$ DP/MAS NMR spectra of (b) the initial $\mathrm{H}^{13} \mathrm{CO}$-tholins (c) and the $400{ }^{\circ} \mathrm{C} \mathrm{H}^{13} \mathrm{CO}$ tholins

Figure 2. Raman spectrum of the $400^{\circ} \mathrm{CH}^{13} \mathrm{CO}$ - tholin. The $\mathrm{G}$ and D bands at $\sim 1537 \mathrm{~cm}^{-1}$ and $\sim 1310 \mathrm{~cm}^{-1}$, respectively reveal a polyaromatic structure typical of coals and kerogens. The low signal-to-noise ratio and spectral artifacts at the edge are due to the high fluorescence background only partly corrected by the baseline correction.

Figure 3. (a) Normalized XANES spectra of the $400{ }^{\circ} \mathrm{C} \mathrm{H}^{13} \mathrm{CO}$ and $\mathrm{H}^{12} \mathrm{CO}$-tholins dominated by aliphatic $\left(-\mathrm{CH}_{\mathrm{x}}\right), \mathrm{C}=\mathrm{C}$ and $\mathrm{C}=\mathrm{O}$ functional groups and (b) XANES and NMR methods correlation of the $400{ }^{\circ} \mathrm{CH}^{13} \mathrm{CO}$-tholins with the re-produced data from Le Guillou et al. (2018) and Le Brech et al. (2016).

Figure 4. Corrected infrared spectra of $\mathrm{H}^{12} \mathrm{CO}$ and $\mathrm{H}^{13} \mathrm{CO}$-tholins samples. Highlighted attributions $\left(\mathrm{CH}_{3}\right.$ and $\mathrm{CH}_{2}$ anti-symmetric modes and $\mathrm{C}=\mathrm{O}$ and $\mathrm{C}=\mathrm{C}$ stretching modes) are those used for quantitative study.

Figure 5. Detailed components of $\mathrm{CH}_{\mathrm{x}}$ mode in different phases (liquid, crystalline, amorphous) in an example of alkane $\mathrm{C}_{20} \mathrm{H}_{42}$ (modified from MacPhail et al., 1984).

Figure 6. (a) Fit of the alkyl band of an IOM extracted from the Orgueil chondrite, obtained through the artificial bees' algorithm. This fit was achieved with the 7 Gaussian model and all components FWHM forced to $35 \mathrm{~cm}^{-1}$. (b)-(c) Illusion of uncertainty of spectral decomposition methods in different scenarios: Gaussian fix a FWHM of $35 \mathrm{~cm}^{-1}$ (Blue); Voigt fix a FWHM of $20-40 \mathrm{~cm}^{-1}$ (Yellow); Gaussian free a FWHM range of $20-40 \mathrm{~cm}^{-1}$ (Green), Voigt free a FWHM range of $20-40 \mathrm{~cm}^{-1}$ (Red).

Figure 7. Normalized spectral intensity of the organic functional groups derived from the calibration of $\mathrm{C}=\mathrm{O}$ and $\mathrm{C}=\mathrm{C}$ and $\mathrm{CH}_{\mathrm{x}}$ groups using the DP/MAS NMR and FTIR calibration for the $400^{\circ} \mathrm{CH}^{13} \mathrm{CO}$ - tholins on (a) Murchison (CM2), (b) Orgueil (CI1), (c) EET 92042 (CR2) and (d) Tagish Lake (C2-ung) IOM in the comparison with the SP/MAS ${ }^{13} \mathrm{C}$ NMR spectra (Cody et al., 2005) and CP/MAS ${ }^{13} \mathrm{C}$ NMR (Gardinier et al., 2000).

Figure 8. Comparisons plots between (a) $\mathrm{n}_{\mathrm{CHx}} / \mathrm{n}_{\mathrm{C}=\mathrm{C}}$ and $\mathrm{n}_{\mathrm{CHx}} / \mathrm{n}_{\mathrm{C}=\mathrm{O}}$ ratios (b) $\mathrm{n}_{\mathrm{CHx}} / \mathrm{n}_{\mathrm{C}=\mathrm{C}}$ and $\mathrm{nc}=\mathrm{o} / \mathrm{n} \mathrm{C}=\mathrm{C}$ of IOM functional C groups (Murchison (CM2), Orgueil (CI1), EET92042 (CR2) and Tagish Lake (C2-ung)) derived from FTIR-NMR quantification and the previous NMR's measurement (Cody \& Alexander, 2005; Gardinier et al., 2000; Yabuta et al., 2005).

Figure 9. Comparisons plots between (a) $\mathrm{H} / \mathrm{C}$ and $\mathrm{O} / \mathrm{C}$ atomic ratios of studied coals (b) Normalized intensity of $\mathrm{C}=\mathrm{O}, \mathrm{n}_{\mathrm{C}=\mathrm{O}}+\mathrm{COOH}_{(\mathrm{R})}$ vs. vitrinite reflectance, (c) $\mathrm{n}_{\mathrm{CH}} / \mathrm{n}_{\mathrm{C}}=\mathrm{C}$ vs. vitrinite reflectance and (d) Calculated $A_{\mathrm{CH}} / \mathrm{A}_{\mathrm{C}=\mathrm{C}}$ vs. vitrinite reflectance derived from FTIR-NMR quantification and the previous NMR's measurement (Wei \& Tang, 2018). 